\title{
Will Customize Appliances Replace Standard Orthodontic Treatment?
}

\author{
K Hero Breuning, ${ }^{1, *}$ \\ ${ }^{1}$ Department of Orthodontics, Radboud University Nijmegen, Nijmegen, The Netherlands \\ "Corresponding author: K Hero Breuning, Department of Orthodontics, Radboud University Nijmegen, Nijmegen, The Netherlands. E-mail: herobreuning@gmail.com
}

Received 2016 February 03; Accepted 2016 February 15.

\begin{abstract}
In this review, new developments as has been recently published such as digital dental models, virtual treatment planning, intra oral scanning, merging of the intraoral scan and the СВCT and finally the use of custom orthodontic brackets and computer bend wires will be discussed.
\end{abstract}

Keywords: Orthodontics, 3D Imaging, Intra Oral Scanning, CBCT, Digital Planning, SETUP

\section{Context}

If orthodontic treatment is indicated, a majority of the orthodontist will use 2D intra and extra oral photographs, an OPT and a headplate for documentation and treatment planning (1). If fixed appliances will be the treatment of choice traditionally straight wire metal or ceramic brackets already available in the orthodontic office, are bonded onto the dentition and a standard set of pre-bend wires are used for initial correction of tooth position. After aligning, the orthodontist decides which bracket needs replacement and finally, the orthodontist will bend finishing wires to complete the case. In this traditional treatment process, the result of treatment and the treatment time needed will be determined by the orthodontist and depends on the skills of the practitioner and off course, the cooperation of the patient.

This process will change as patients, referring dentists and insurance companies demand predictable results and a predictable treatment time. Referring dentists and their patients expect optimal esthetic and functional improvement of the malocclusion. Reduction of treatment time, treatment cost and life time stability should also be achieved. Especially the adult patient demands to be treated with invisible orthodontic appliances. About four decades ago, 2D radiographic images such as headplates were used for treatment planning (2). Currently, 3D visual treatment objective software, such as Dolphin and Anatomage, is available to plan orthodontic (and surgical) treatment and show the selected treatment procedure, the orthodontic appliances required and the planned final result to the patient, before the start of treatment.

For difficult cases, such as replacement of teeth by implants and surgical procedures, visualization of treatment using dental setups in wax and a surgical planning with plaster models and articulators are used in most orthodontic offices. The OrthoCad company, started to digitize plaster models of the dentition in 1999. These digital dental models can be used for digital dental set ups for virtual treatment. Virtual placement of orthodontic brackets on the setup is possible and this position can be transferred to the pre-treatment model to enable efficient bonding of brackets using indirect bonding trays. The increase in the use of digital dental models all over the world shows, that these digital dental models could replace plaster casts (3). Laser scanners or CT radiographs can also be used for the scanning of dental models or dental impressions to make digital models. To replace dental impressions and plaster models, intra-oral scanners can be used (4). These dental (intra-oral) scans should be transformed into standard tessellation language (STL) files. This file format is supported by many software packages and is widely used for rapid prototyping and computer-aided manufacturing. Intra oral color scanners such as the Trios (3 shape company Denmark), can be used to make a intra oral color scan of the dentition, the palate and the alveolar bone in a relative short acquisition time and these images can be used as a replacement of intra oral photographs (Figure 1).

\section{Evidence Acquisition}

Once the files of a digital dental model are available, image segmentation (the process of partitioning a digital image into multiple segments) can be used to locate and separate the dental crowns out of the digital dental model. The resulting dental crown contours after image segmentation can be used to create $3 \mathrm{D}$ reconstructions with the 


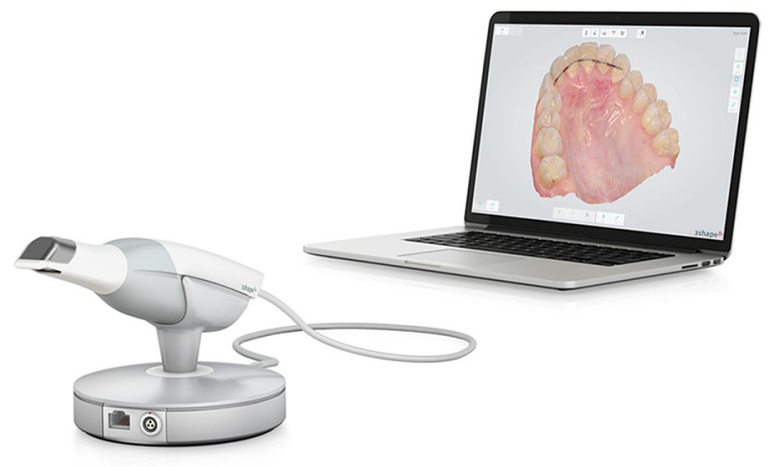

Figure 1. Intraoral Color Scans Can Replace Intra Oral Photographs

help of interpolation algorithms. Different treatment possibilities can be evaluated in minutes. Smile design used by dentists is now also possible with the newest orthodontic treatment planning software. New developments such as the combination of orthodontic, dental and maxillofacial software programs to plan the optimal esthetic and functional optimal outcome will further increase the predictability of treatment outcome.

The digital imaging and communications in medicine (DICOM) files of 3D radiographs of a patient's head, from a cone beam computer tomography (CBCT) radiograph can also be transformed into STL files. Accurate superimposition of an intra oral scan and a CBCT can now be used to increase the accuracy of the dentition on the CBCT. Recent CBCT machines such as the Planmeca CBCT machine, can be used for making 2D and 3D radiographic images and additional to the radiographs, 3D facial photographs can be made at the same time. These facial photographs can replace traditional 2D photographs. However, according to the guidelines for CBCT's these radiographs should not be taken for every patient and these radiographs should replace traditional 2D radiographic images (5).

The use of virtual articulators for evaluation of the articulation of the digital models and the setup as well as 3D jaw movement registration devices such as te Sicat registration device can be used to superimposed the registration on the CBCT images. Accurate digital registration of the occlusion with devices such as the T scan, can be used for further improvement of the quality of treatment planning and evaluation of the occlusion after treatment.

\subsection{Fabrication of a Dental Setup}

After segmentation, the teeth (including the dental roots), can be used to make a setup of the dentition to simulate orthodontic treatment. Recently, the Suresmile company accepts also CBCT radiographs (with a voxel size of
$0.3 \mathrm{~mm}$ ), for making a setup of the dentition including the dental roots. Simulation of treatment is now possible in 3 dimensions and in a $1: 1$ ratio. As information of the dimensions and quality of the alveolar bone is available from the CBCT images, this information can be used for improved planning of the desired tooth position after treatment. The first company, who successfully introduced a process of virtual orthodontic treatment using a dental set up on digital dental models for fabrication of custom orthodontic appliances (aligners), was Invisalign. This company still uses virtual tooth movement on digital dental models for fabrication of a set of 3D printed models. A series of aligner trays made on the printed dental models, can be used to move teeth in steps into the desired direction. Aligner 1 is used to change tooth position from the initial tooth position to the planned tooth position (stage 1 ) and then tray 2 should be used to guide the tooth position from stage 1 to 2 and so on. In the beginning, the indication for aligner treatment to move teeth was limited. Improvements in aligner material and the use of acrylic attachments attached to the dentition extended the indication for aligner appliances. It is now possible to treat more severe dental malocclusions with aligners.

Of course, the steps as made in the virtual treatment planning should be realistic. Essential for successful use of every orthodontic appliance is knowledge of tooth movement and its restrictions. The dental technician should therefore not indicate the treatment needed for a specific case. In a total digital workflow for orthodontic treatment, the orthodontist should make a treatment plan based on the documentation and the wishes of the patient and then send the dental impressions, plaster model or an intra-oral scan to a dental lab for segmentation and fabrication of an initial setup according to his treatment plan (Figure 2).

The orthodontist should then evaluate this virtual treatment plan. If required, corrections are indicated by the orthodontist and returned to the orthodontic lab. Ideally, the orthodontist should have a software program to correct the initial setup. However, it takes a lot of time to master those software programs so to reduce the time the orthodontist has to spend behind the computer a lab technician will make the setup. After approval of the virtual treatment plan by the orthodontist and the patient, orthodontic appliances should be ordered. If fixed appliances are selected, scanned standard brackets can be positioned with dedicated software on the original dental model or the setup by the dental technician. Again, the orthodontist should evaluate the bracket position (Figure 3 ).

After approval of the bracket position, an indirect bonding tray can be fabricated on 3D printed models or an indirect bonding tray can be designed and printed directly with dedicated software. 


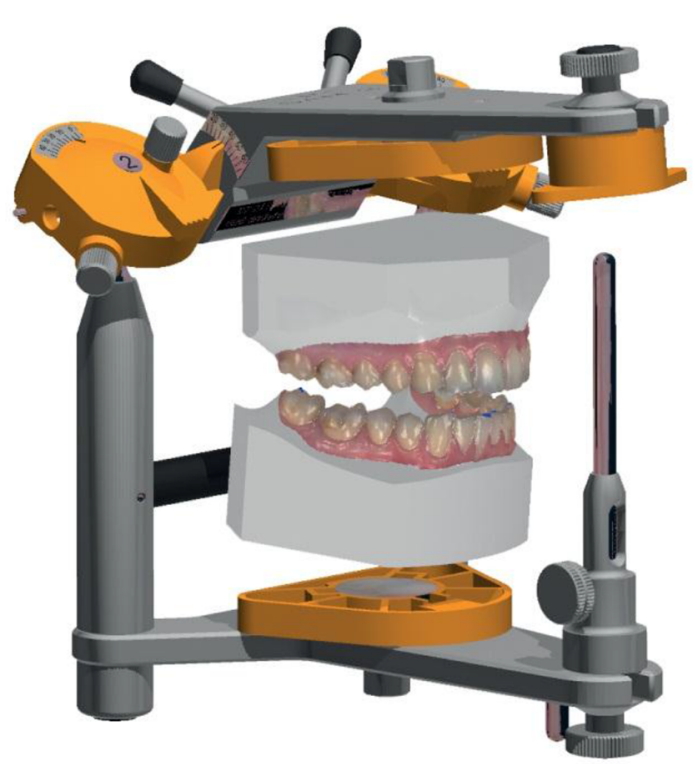

Figure 2. Dental Setup

\subsection{Custom Orthodontic Bracket Systems}

Custom buccal or custom lingual appliances or a set of aligners with attachments can be fabricated according to the instructions of the orthodontist. An increasing amount of companies use designing software to design custom orthodontic appliances according to the wishes of the orthodontist and the patient. The use of an indirect bonding tray for accurate bracket placement and custom orthodontic wires bend by a wire bending robot will increase treatment efficiency. A popular custom system, Insignia, an Ormco system has been introduced for treating orthodontic patients with custom buccal brackets. Insignia delivers a custom solution for a range of orthodontic brackets placed on the buccal surface of the dentition. (DamonQ, Damon clear and Inspire Ice brackets). Insignia uses a very nice virtual planning system, which shows the limits of the dental arch, because the outline of the mandibular alveolar can be evaluated in the setup. Furthermore, the occlusion of the digital planning in this system can be evaluated. The base of the brackets can be individualized and the virtual bracket placement can be transferred to the dentition using indirect-bonding transfer jigs. After approval of the setup, an indirect bonding tray containing the brackets as selected by the orthodontist is shipped to the orthodontic office. Orthodontic treatment starts with indirect bonding of the brackets. Reduction of chair time used for placement and replacement of brackets and the bending of (finishing) wires can be ex- pected. Flexible round or rectangular wires are then used during 3 to 5 months for initial leveling and aligning. A progress intra oral scan or a progress CBCT of the patient is then used to evaluate treatment progress and if needed, to make a second dental set up. After approval of this follow up setup, custom wires are bent by a wire bending robot, or a new set of aligners are fabricated for further correction of the tooth position. If a follow up CBCT is used, the position of the dental roots and the available alveolar bone can be visualized and corrected in a setup. The use of 3D radiographic images such as CBCT's for treatment planning and evaluation also enhances an orthodontist's ability to collaborate with oral surgeons and dentists by providing an interactive data model that can be used for surgical and restorative planning. An important feature of customized orthodontic appliance systems is the fabrication of a set of aligners to finish cases started with fixed appliances (Hybrid orthodontic appliances). Major draw backs of custom orthodontic appliance systems are the costs of these systems.

As an alternative for custom aligner systems and custom buccal orthodontic appliances, several custom lingual fixed appliance systems have been introduced. A popular system for custom lingual brackets is Incognito (3M, Unitek). Recently, alternative custom lingual systems such as harmony and e-Brace/eLock have been introduced (Figure 4).

The bending of finishing wires for lingual cases is difficult so custom bended wires can be more efficient. If correction of tooth position during treatment is needed, because of side effects during correction or individual responses to orthodontic forces, orthodontic treatment should be reevaluated, during treatment. The data of this reevaluation (impressions or intra oral scans) can be send to the orthodontic lab for a follow up set up and fabrication of individual wires to finish the lingual case.

The costs of all these custom orthodontic appliances are much higher compared to the traditional fixed appliance systems. So there should be advantages for both the patient and the orthodontist to compensate this price difference. Some of the advantages for these custom orthodontic appliances mentioned in advertisements and in the orthodontic literature are: 1 , digital dental models for easy retrieval, accurate measurements because clipping and zooming functions can be used; 2 , no space is needed for plaster storage, the models are unbreakable, can be transported by internet, copies of the models will be available; 3 , segmentation of the dentition enables virtual tooth movement (dental setup) and custom orthodontic appliances can be fabricated using digital dental images; 4 , digital dental models can be superimposed for evaluation of tooth movement or growth; 5 , a digital setup of treatment 


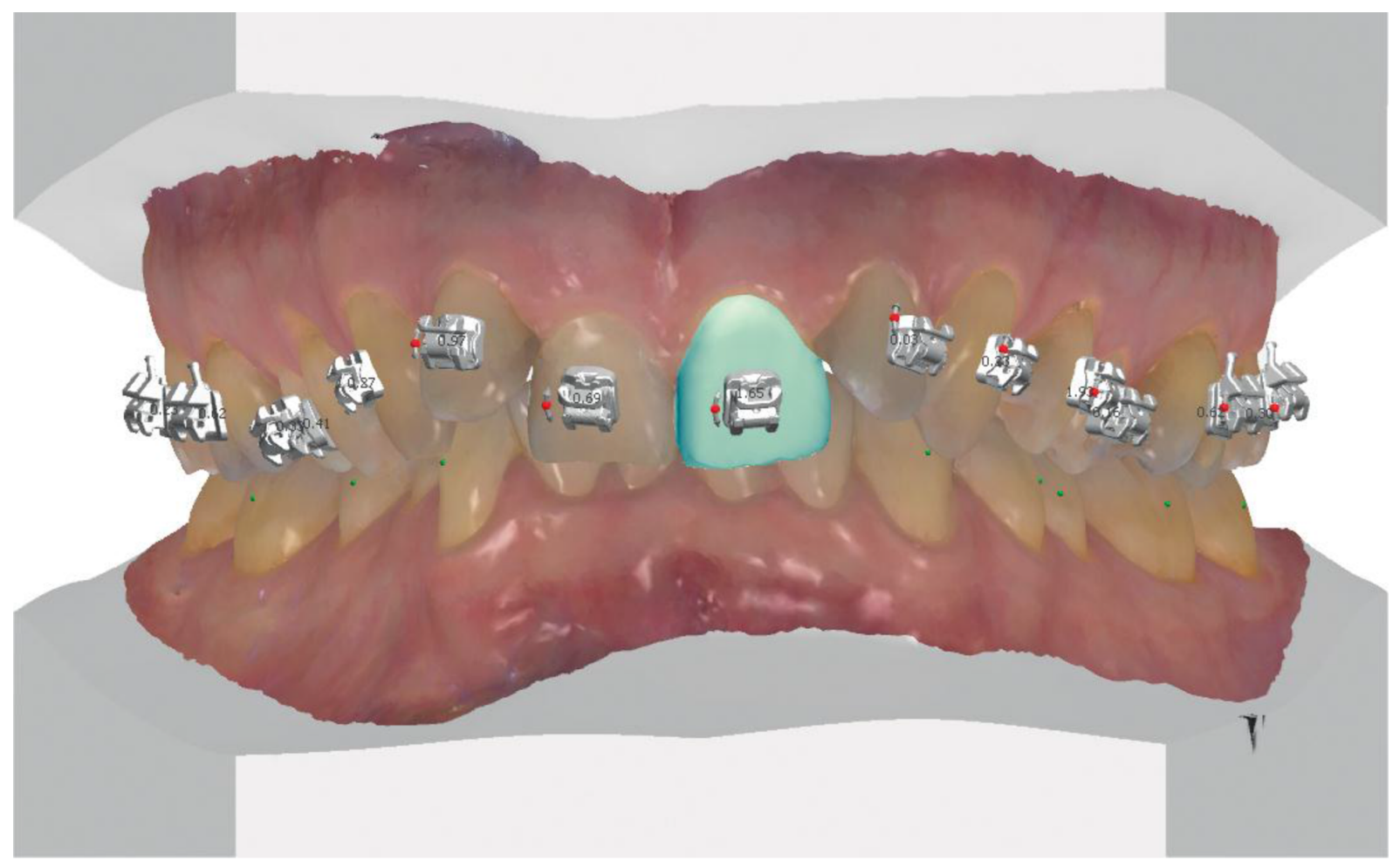

Figure 3. Virtual Bracket Positioning

is available for every case. Alternative treatment plans can be evaluated and discussed with the patient; 6 , communication with the dentist and the oral surgeon in the evaluation of the virtual treatment plan before the start of treatment is easy; 7, if needed, an alternative treatment plan and appliances used can be discussed with colleagues on line; 8 , the use of customized appliances should lead to shorter treatment and improved results and the finishing phase of orthodontic treatment should be shorter because repositioning of brackets and bending of finishing wires is no longer needed; 9, superimposition of digital images is possible to monitor and evaluate the result of treatment.

Off course, research is needed to evaluate the efficiency of these custom appliances systems. In a systematic review of the literature on the Invisalign system (6), were unable to draw strong conclusions about the effectiveness of this system.

In their review, they emphasized the need for randomized clinical trials to compare the effectiveness of this system compared to alternative treatment systems (7), concluded that Invisalign was especially deficient in its ability to correct large anteroposterior discrepancies and occlusal contacts. They felt that the strengths of Invisalign were its ability to close spaces and correct anterior rota- tions and marginal ridge heights. they added Invisalign patients finished 4 months sooner than those with fixed appliances on average. Kravitz et al. (8) published a prospective clinical study evaluating the efficacy of tooth movement with Invisalign concluded: We still have much to learn regarding the biomechanics and efficacy of the Invisalign system. A better understanding of Invisalign's ability to move teeth might help the clinician select suitable patients for treatment, guides the proper sequencing of movement, and reduces the need for case refinement. In a recent study, by Buschang (9), which was published only on line, a selection of 150 patients was treated with conventional edgewise were compared to 150 patients treated with aligners. These authors concluded that conventional Edgewise treatment required significantly more visits (approximately 4), a longer treatment duration (5.5 months) and more emergency visits (1) compared to aligner treatment. The patients in these two groups did have a Class I molar and a class I cuspid relation and only minor crowding and an overjet less than $4 \mathrm{~mm}$ before the beginning of treatment. It was mentioned that the aligner treatment was significantly more expensive.

The SureSmile system claims, that this system will not only reduce treatment time, but should also increase treat- 
A

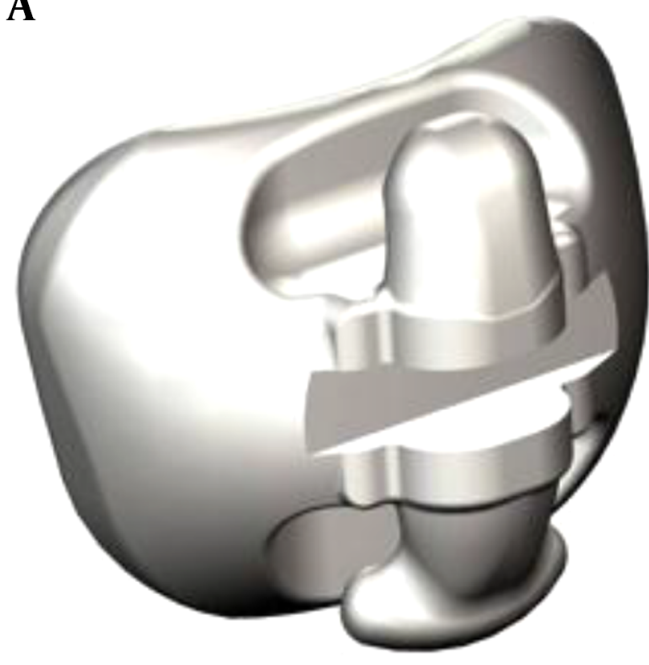

B

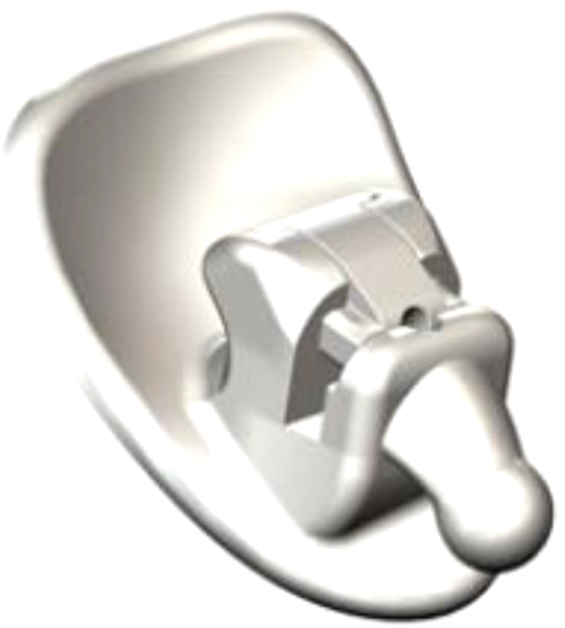

C

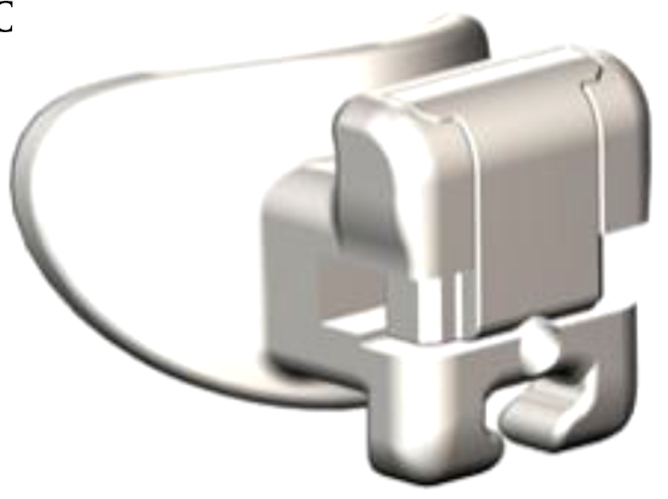

Figure 4. A shows eBrace, B and C show the eLock bracket.

ment result. For evaluation of treatment result and treatment time randomized clinical trials are needed. These studies are not available for SureSmile. According to Saxe and colleagues (10) used the ABO and the OGB (objective grading system) system to evaluate treatment quality. It was reported that SureSimile patients had on average, lower scores and a shorter treatment time compared to the patients treated with conventional orthodontic appliances. However; the research protocol for this study was insufficient and the conclusions are therefore not scientifically proven. In the study published by Altford and colleagues (11), the inclusion and exclusion criteria of both groups (63 cases conventional and 69 cases treated with Suresmile) were specified. Differences in age, sex and discrepancy index of the malocclusion (DI scores) before the start of treatment were compared. After treatment ABO and CRE scores (radiographic evaluation), revealed, that the Suresmile group had significant lower DI scores. The root angulation variable in the SureSmile group tended to be lower. Treatment time for the Suresmile patients was significantly shorter. 31\% (7.2 months) and the result increased with $11 \%$. Larson and colleagues (12) evaluated the effectiveness of Suresmile treatment by superimposition of the digital planned end result and the result achieved. 
These researchers concluded that Mesial-distal tooth position was clinically ideal for all teeth with the exception of maxillary lateral incisors and second molars. Faciallingual tooth position was clinically ideal for all teeth except maxillary central incisors, premolars, and molars, and mandibular incisors and second molars. Vertical tooth position was clinically ideal for all teeth except mandibular second molars. For crown torque, tip, and rotation, discrepancy exceeded the limits considered clinically ideal for all teeth except for crown torque on mandibular second premolars and crown tip on mandibular second premolars and first molars. These researchers concluded: together with the findings of the present study, these results suggest that utilization of computer-assisted treatment technology during comprehensive orthodontic treatment can achieve results equivalent or superior to conventional fixed appliance treatment. Clinicians are encouraged to utilize the knowledge of dimensions in which the final tooth position is less consistent with the predicted position to build compensations into the virtual treatment plan.

According to Sachdeva and colleagues (13) who is not independent from the Suresmile company, published an article in which a large group of patients (9390 patients treated with Suresmile and 2945 patients treated with conventional orthodontic appliances) in 142 orthodontic offices were compared. In the pooled results, a significant reduction in treatment time was reported. Again, research methods in this study are questionable.

The efficiency of the Incognito system to achieve the planned treatment outcome has been evaluated by Grauer and Proffit (14). These researchers evaluated digital models made from the set up in wax of 94 consecutive patients from 1 practice, representing a broad range of orthodontic problems, and digital casts from the final models for each patient registered individually for the maxillary and mandibular dental arches. Individual tooth discrepancies between the setup and actual outcome were computed and expressed in terms of a six degrees of freedom rectangular coordinate system. They concluded: these fully customized lingual orthodontic appliances were accurate in achieving the goals planned at the initial setup, except for the full amount of planned expansion and the inclination at the second molars. In this study, a difference in position of more than $1 \mathrm{~mm}$ or more than $4^{\circ}$ was considered inaccurate. In this study, only the upper and lower arch and not the final occlusion were evaluated.

The effectiveness for the Insignia system was studied by Weber and colleagues (15). In this pilot study, it was found, that initial PAR scores, after adjusting for age at start of treatment and initial PAR, were not significantly different between the two groups, which showed a similar re- duction in PAR scores after treatment. Because the interaction between the age at start of treatment and the treatment group was statistically significant, the $\mathrm{ABO}$ score for each group was estimated and compared at age 15 and the overall mean age of 26 . At both ages, the Insignia group had a lower average $\mathrm{ABO}$ score, indicating a finished result closer to the ideal ABO criteria the efficiency of the Insignia system was in this study also evaluated. It was concluded, that the adjusted treatment time was significantly shorter for the Insignia patients, with about seven fewer appointments on average. Numbers of unscheduled emergency appointments, debonded brackets, repositioned brackets, and wire bends were similar between the two treatment groups.

In summary, traditional planning for orthodontic treatment using a plaster cast, 2D photographs, a lateral headplate and an OPT radiograph seems to become outdated. These images will be replaced by 3D extra and intra oral images of the face of the patient. As evaluation of function, movement and aesthetics is indicated, movement will also be recorded. The use of a CBCT with a Field of View limited to the dental arches, with a reduced scanning time could become an alternative for $2 \mathrm{D}$ radiographs (16). Intra oral scanning of the dentition and the alveolar process can be used to increase the accuracy of the dentition on CBCT images, the intraoral scans can also be easily transferred to an orthodontic lab for making a digital dental setup.

It is the orthodontist who should learn how to transfer a treatment plan for a specific patient into a virtual treatment plan. This plan should be presented to the patient. Depending on the choice of the patient, aligners, custom lingual fixed appliances or custom buccal fixed appliances or a combination of fixed and removable custom appliances could then be selected for the actual treatment. The files of the virtual planning (STL files) will be sent to an orthodontic lab for fabrication of these appliances. It can be expected, that in near future, only 3D printers and bending robots will be used for fabrication of custom orthodontic appliances. Because of the individual tooth movements during orthodontic treatment, it is not realistic to plan an orthodontic treatment from A to Z. Even if custom appliance systems are used, side effects of orthodontic treatment mechanics will reduce the accuracy of the outcome of orthodontic treatment. These side effects as caused by orthodontic treatment mechanics such as class II elastics can be inevitable. So, at least one refinement of the treatment plan during treatment will be needed. After an update scan during treatment, the next set of aligners or the next wire(s) for fixed appliance treatment will be delivered by the company. Only a well experienced orthodontist could incorporate some over corrections to the initial vir- 
tual planning to anticipate on the side effects of orthodontic mechanics and will be able to achieve the planned result without a refinement during treatment.

Is the orthodontist still in control of treatment if custom appliances are used? In my opinion, the orthodontist will have total control over the treatment of a specific patient when digital treatment planning and custom appliances are used. Therefore, it is essential that planning and monitoring of the orthodontic treatment will remain a task of a trained orthodontist and is not managed by a technician in an orthodontic lab. Currently, some systems send the digital set up to the orthodontist and provide a viewer only. The orthodontist should send their remarks to the company. The lab will then correct the set up as indicated by the orthodontist. Other systems enable the orthodontist to correct the setup, using their own computer. This corrected set up is transferred to the orthodontic lab by email and then used for appliance fabrication according to the final treatment plan of the orthodontist.

Because indirect bonding, prefabricated orthodontic appliances, custom wires or aligners are used, the role of the orthodontist during appliance placement and the process of changing wires or aligners during treatment can possibly be reduced. But in my opinion, the use of digital planning for orthodontic treatment and custom appliances for orthodontic treatment will grow. The price of individualized appliances will be reduced and in the end, effective orthodontic treatment leading to high quality results will be available for a large group of patients.

\section{Results}

Form the literature it is clear, that these new developments will finally enable to use a virtual head in 3D for effective treatment planning. New developments, such as robots to bend wires and customized design of orthodontic appliances with new fabrication techniques such as 3D printing will enable to show the patient a preview of the expected result. Furthermore, the custom appliances will increase treatment efficiency, reduce treatment time and the final result will be improved.

\section{Conclusions}

A complete digital workflow for orthodontic documentation, treatment planning, appliance design, appliance fabrication, treatment evaluation during and after treatment will soon replace the traditional workflow in orthodontics. Intra oral color scanners will replace impressions and intra-oral photographs. CBCT machines which can be also used to make 3D facial scans, will be used to will replace the procedure to make traditional 2D radiograph and 2D extra-oral photographs.

\subsection{Companies Mentioned}

Anatomage: San Jose, California, USA; Dolphin imaging and software solutions: Chatsworth, California, USA; Harmony, (American orthodontics): Sheboygan, Wisconsin, USA; Incognito (3M/Unitek): Monrovia, California, USA; Insignia: (Ormco Corporation), Orange, California, USA; Sicat: Sirona Dental GmbH Sirona Dental GmbH Wals bei Salzburg Osterreich; T-scan: Tekscan, Boston, USA; OrthoCad: (align technology, Inc.) San Jose, California, USA; Suresmile: Orametrix, Inc. Richardson, Texas, USA.

\section{References}

1. Rischen RJ, Breuning $\mathrm{KH}$, Bronkhorst EM, Kuijpers-Jagtman AM. Records needed for orthodontic diagnosis and treatment planning: a systematic review. PLoS One. 2013;8(11):e74186. doi: 10.1371/journal.pone.0074186. [PubMed: 24265669].

2. Bench RW, Gugino CF, Hilgers JJ. Bio-progressive therapy. 3. J Clin Orthod. 1977;11(11):744-63. [PubMed: 273601].

3. Peluso MJ, Josell SD, Levine SW, Lorei BJ. Digital models: An introduction. Seminars in Orthodontics. 2004;10(3):226-38. doi: 10.1053/j.sodo.2004.05.007.

4. Garino F, Garino B, Castroflorio T. The iTero intraoral scanner in Invisalign treatment: a two-year report. Clin Oral Implants Res. 2014;48:98-106.

5. Position statement by the American Academy of Oral and Maxillofacial Radiology, American Academy of Oral and Maxillofacial Radiology. Clinical recommendations regarding use of cone beam computed tomography in orthodontics. Oral Surg Oral Med Oral Pathol Oral Radio. 2013 Aug;116(2):238-57. doi:10.1016/j.00oo.2013.06.002.

6. Lagravere MO, Flores-Mir C. The treatment effects of Invisalign orthodontic aligners: a systematic review. J Am Dent Assoc. 2005;136(12):1724-9. [PubMed: 16383056].

7. Djeu G, Shelton C, Maganzini A. Outcome assessment of Invisalign and traditional orthodontic treatment compared with the American Board of Orthodontics objective grading system. Am J Orthod Dentofacial Orthop. 2005;128(3):292-8. doi: 10.1016/j.ajodo.2005.06.002. [PubMed: 16168325] discussion 298.

8. Kravitz ND, Kusnoto B, BeGole E, Obrez A, Agran B. How well does Invisalign work? A prospective clinical study evaluating the efficacy of tooth movement with Invisalign. Am J Orthod Dentofacial Orthop. 2009;135(1):27-35. doi: 10.1016/j.ajodo.2007.05.018. [PubMed: 19121497].

9. Buschang PH, Shaw SG, Ross M, Crosby D, Campbell P. Comparative time efficiency of aligner therapy and conventional edgewise braces. The Angle Orthodontist. 2013:131118094755008. doi: 10.2319/062113466.1.

10. Saxe AK, Louie LJ, Mah J. Efficiency and effectiveness of SureSmile. World J Orthod. 2010;11(1):16-22. [PubMed: 20209172].

11. Alford TJ, Roberts WE, Hartsfield JJ, Eckert GJ, Snyder RJ. Clinical outcomes for patients finished with the SureSmile method compared with conventional fixed orthodontic therapy. Angle Orthod. 2011;81(3):383-8. doi: 10.2319/071810-413.1. [PubMed: 21261488].

12. Larson BE, Vaubel CJ, Grunheid T. Effectiveness of computer-assisted orthodontic treatment technology to achieve predicted outcomes. Angle Orthod. 2013;83(4):557-62. doi: 10.2319/080612-635.1. [PubMed: 23181776]. 
13. Sachdeva RCL, Aranha S, Egan ME, Gross HT, Sachdeva NS, Frans C. G. , et al. Treatment time: SureSmile vs conventional. Orthodontics. 2012;13:72.

14. Grauer D, Proffit WR. Accuracy in tooth positioning with a fully customized lingual orthodontic appliance. Am J Orthod Dentofacial Orthop. 2011;140(3):433-43. doi: 10.1016/j.ajodo.2011.01.020. [PubMed: 21889089].

15. Weber DJ, Koroluk LD, Phillips C, Nguyen T, Proffit WR. Clinical effec- tiveness and efficiency of customized vs. conventional preadjusted bracket systems. J Clin Orthod. 2013;47(4):261-6. [PubMed: 23660822] quiz 268.

16. Ludlow JB, Walker C. Assessment of phantom dosimetry and image quality of i-CAT FLX cone-beam computed tomography. Am J Orthod Dentofacial Orthop. 2013;144(6):802-17. doi:10.1016/j.ajodo.2013.07.013. [PubMed: 24286904]. 